\title{
Acousto-optic polarization switch with parallax compensation
}

\author{
Boichuk N. A., "Pilgun Yu. V. and Smirnov Ye. M.
}

Faculty of Radiophysics, Electronics and Computer Systems, Taras Shevchenko National University of Kyiv, 64/13 Volodymyrska Street, 01601 Kyiv, Ukraine; *e-mail: ply@univ.kiev.ua

Received: 26.01 .2018

\begin{abstract}
We propose acousto-optic (AO) polarization switch based on a paratellurite crystal. The geometry of anisotropic interaction is chosen so as to satisfy the Bragg conditions for the two alternative light polarizations at the wavelength $785 \mathrm{~nm}$. For this both the direct and reflected from crystal face acoustic waves are used. The polarization of the output light is governed by switching between one of the two alternative frequencies of a driving ultrasound signal applied to the $\mathrm{AO}$ cell. The device is parallax-compensated, so that the light beams with different polarizations leave the cell at the same point, with no transverse shift, and propagate along the same direction. A corresponding AO polarization switch is manufactured and its characteristics are studied experimentally. The measured contrast ratio of the device is better than 1800:1.
\end{abstract}

Keywords: acousto-optic diffraction, acousto-optic switches, anisotropic Bragg diffraction, linear polarization, polarization modulator, paratellurite, tellurium dioxide.

PACS: $78.20 . \mathrm{Hp}, 42.79 . \mathrm{Jq}$

UDC: $535.42,534.522 .1$

\section{Introduction}

Possibilities for creating acousto-optic (AO) polarization modulators have been demonstrated in Refs. $[1,2]$. The authors of these works have described a device with two piezoelectric transducers attached to the faces of paratellurite sample oriented at different angles to the crystallographic direction [110]. This geometry of ultrasonic transducers makes the device difficult to manufacture and complicates the corresponding driving electronics. On the other hand, one can recall AO devices in which the light diffracts at the ultrasound wave reflected from a free crystal boundary [3, 4]. The idea of using reflected acoustic waves benefits from avoiding two separate transducers. In line with this idea, in the present work we develop an AO polarization switch that operates at the light wavelength $785 \mathrm{~nm}$. In particular, we analyze the possibilities for satisfying simultaneously the Bragg conditions for the two light polarizations and using a single transducer to control them. We also study the highest contrast ratio achievable for the appropriate device made of paratellurite.

\section{Simultaneous diffraction of different light polarizations}

Paratellurite represents a important material for creating AO devices because it requires relatively low ultrasound powers due to its high AO figure of merit [5]. The most used configuration is based on the off-axis anisotropic Bragg diffraction [6]. However, high diffraction efficiencies (above $90 \%$ ) in this configuration are achieved only for completely polarized light. When controlling depolarized light or light of a 'mixed' polarization, the input-light polarization should be treated as a sum of two alternative orthogonal polarizations, and two different AO-interaction processes should be considered for each polarization mode. In crystals, these two polarizations correspond to 
optical eigenwaves propagating along a selected direction. Due to optical birefringence, the incident light is split into two rays at the entrance face of crystal. Although the ordinary and extraordinary rays propagate along slightly different directions inside a crystal, we will ignore this fact in our preliminary discussion of the interaction geometry and address this issue later on. It is justifiable since, for the common interaction geometries, the incident light propagates very close to the optic axis, so that the difference in the refractive indices for the ordinary and extraordinary rays is small enough.

In the same way as in the works by Antonov [1, 2], we consider the AO interaction of an arbitrarily polarized light at two acoustic waves propagating along different directions (see Fig. 1). The Bragg conditions are satisfied simultaneously for the both polarizations of light that propagates at some angle $\theta_{i}$ with respect to the optic axis. Moreover, the interaction geometry is selected so that it makes the diffraction angles $\theta_{d}$ the same for the both diffracted waves. The wave vectors of the incident light are denoted as $\mathbf{k}_{i o}$ and $\mathbf{k}_{i e}$, where the subscripts " $o$ " and " $e$ " correspond to the ordinary and extraordinary rays, respectively. The corresponding diffracted wave vectors are denoted as $\mathbf{k}_{d o}$ and $\mathbf{k}_{d e}$. We will consider only anisotropic interaction which is accompanied with polarization changes, $\mathbf{k}_{i o} \rightarrow \mathbf{k}_{d e}$ or $\mathbf{k}_{i e} \rightarrow \mathbf{k}_{d o}$. We refer to these diffraction types as "oe" or "eo" in the rest of the text.

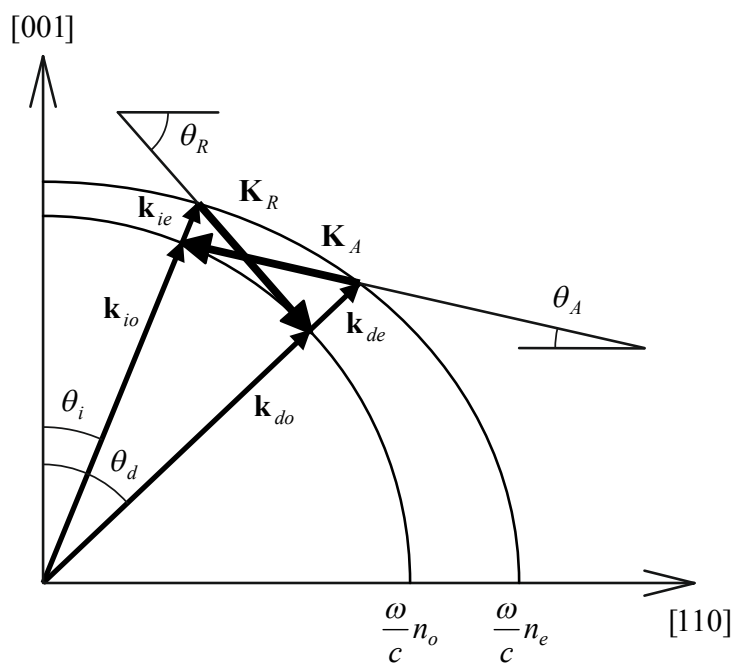

Fig. 1. Wave vector diagram of $A O$ interaction with arbitrarily polarized light. The Bragg conditions are satisfied due to utilization of two different acoustic waves.

To make the diffraction possible, the momentum-conservation conditions should be simultaneously satisfied for the both light polarizations. This leads to the two independent interaction processes at the two acoustic waves with the wave vectors $\mathbf{K}_{A}$ and $\mathbf{K}_{R}$ :

$$
\mathbf{k}_{d e}=\mathbf{k}_{i o}-\mathbf{K}_{A}, \mathbf{k}_{d o}=\mathbf{k}_{i e}+\mathbf{K}_{R} .
$$

According to our approach, a single acoustic wave, with the wave vector $\mathbf{K}_{A}$, is excited by a piezoelectric transducer, while the second acoustic wave, with the wave vector $\mathbf{K}_{R}$, is obtained as a result of reflection of the first wave from a crystal boundary. Due to the opposite orientation of the involved acoustic waves, the signs of the Doppler shift for the frequency of light wave are also 
different for the two processes:

$$
\omega_{d e}=\omega_{0}-\Omega_{A}, \omega_{d o}=\omega_{0}+\Omega_{R},
$$

where $\omega_{0}=2 \pi \mathrm{c} / \lambda_{0}$ is the optical frequency of the incident light, of which wavelength is equal to $\lambda_{0}$. Finally, the ultrasound frequency required for exciting the acoustic wave with a given wavevector length is related to the ultrasound velocity along the given propagation direction as

$$
\frac{\Omega_{A}}{V\left(\theta_{A}\right)}=\left|\mathbf{K}_{A}\right|, \frac{\Omega_{R}}{V\left(\theta_{R}\right)}=\left|\mathbf{K}_{R}\right| .
$$

\section{Shear acoustic-wave velocity and refractive indices in paratellurite}

To calculate the interaction geometry according to Eqs. (1), we need to express orientation dependences of the ultrasound velocity and the refractive indices in the interaction plane. The relevant dependences of the ultrasound velocity can be derived from the Christoffel equation. For the slow shear wave propagating in the plane $(1 \overline{1} 0)$, the angular dependence of the phase velocity is given by [7]

$$
V\left(\theta_{A}\right)=\sqrt{\frac{1}{\rho}\left(\frac{c_{11}-c_{12}}{2} \cos ^{2} \theta_{A}+c_{44} \sin ^{2} \theta_{A}\right)},
$$

where $\theta_{A}$ is the angle of the wave normal measured from the [110] axis, $\rho=5990 \mathrm{~kg} / \mathrm{m}^{3}$ denotes the material density, and $c_{\lambda \mu}$ are the components of the stiffness tensor in contracted notation $\left(c_{11}=5.57 \times 10^{10} \mathrm{~N} / \mathrm{m}^{2}, c_{12}=5.12 \times 10^{10} \mathrm{~N} / \mathrm{m}^{2}\right.$ and $c_{44}=2.65 \times 10^{10} \mathrm{~N} / \mathrm{m}^{2}$ [8]). Finally, the walkoff angle for the group velocity can be obtained from the phase-velocity dependence [9]:

$$
\tan \psi_{A}=\frac{1}{V\left(\theta_{A}\right)} \frac{\partial V\left(\theta_{A}\right)}{\partial \theta_{A}} .
$$

Paratellurite is an optically active, uniaxial material. Its refractive indices can be found from the equation [10]

$$
\left(n^{2}-n_{10}^{2}\right)\left(n^{2}-n_{20}^{2}\right)=G^{2},
$$

where $n_{10}$ and $n_{20}$ are the refractive indices of extraordinary and ordinary rays determined in the hypothetic case if there were no optical activity, and $G$ is the gyration coefficient. The solutions of Eq. (6) for the propagation directions inclined at some angle $\theta$ from the optic axis are as follows [7]:

$$
\begin{aligned}
& n_{1,2}=\frac{1}{\sqrt{2}} \sqrt{n_{10}^{2}+n_{20}^{2} \pm \sqrt{\left(n_{20}^{2}-n_{10}^{2}\right)^{2}+4 G^{2}}}, \\
& n_{20}(\theta)=n_{o}, n_{10}(\theta)=\frac{n_{e} n_{o}}{\sqrt{n_{e}^{2} \cos ^{2} \theta+n_{o}^{2} \sin ^{2} \theta}}, \\
& G(\theta)=g_{33} \cos ^{2} \theta+g_{11} \sin ^{2} \theta,
\end{aligned}
$$

where $n_{o}$ and $n_{e}$ are the refractive indices of ordinary and extraordinary rays for the direction perpendicular to the optic axis, and $g_{33}$ and $g_{11}$ the gyration tensor components that define the optical rotatory powers along and perpendicular to the optic axis, respectively. The influence of $g_{11}$ for the case of paratellurite is negligible [7], so one can use the approximation $g_{11}=0$. The gyration component $g_{33}$ is linked to the rotatory power $R$ via the relation 


$$
g_{33}=\Delta n_{33} n_{o}=\frac{\lambda n_{o}}{\pi} R
$$

The optical material constants $n_{o}, n_{e}$ and $R$ can be obtained using a two-oscillator model described in Ref. [11]. For the light wavelength $\lambda_{0}=785 \mathrm{~nm}$, the calculated values of these parameters are $n_{o}=2.22833, n_{e}=2.37585$ and $R=50.72 \circ / \mathrm{mm}=885.3 \mathrm{rad} / \mathrm{m}$. The difference in the refractive indices along the optic axis for the right- and left-handed circular light polarizations is given by $\Delta n_{33}=n_{r}-n_{l}=0.00022$. Then the ellipticity of the optical waves reads as (see Ref. [12])

$$
\xi(\theta)=\frac{\Delta n_{a}}{\Delta n_{b}+\sqrt{\Delta n_{b}{ }^{2}+\Delta n_{a}{ }^{2}}},
$$

where $\Delta n_{a}=\left(n_{r}-n_{l}\right) \cos ^{2} \theta$ and $\Delta n_{b}=\left(n_{e}-n_{o}\right) \sin ^{2} \theta$.

Note that, for convenience, the angle $\theta$ that determines the optical parameters is measured with respect to the optic axis [001], whereas the angle $\theta_{A}$ referred to the acoustic properties is measured with respect to the [110] axis. With such definitions, the angles of light and ultrasound propagations are close to each other, i.e. we have $\theta_{i} \sim \theta_{A}$ and $\theta_{d} \sim \theta_{R}$.

\section{Construction of our AO device}

A general design of experimental device used in the current work is depicted in Fig. 2. Slow shear acoustic waves travelling close to the [110] axis in paratellurite experience a significant energy walk-off, which is one of the main factors that influences the design of any actual AO device and, in particular, the selection of size of a crystal plate.

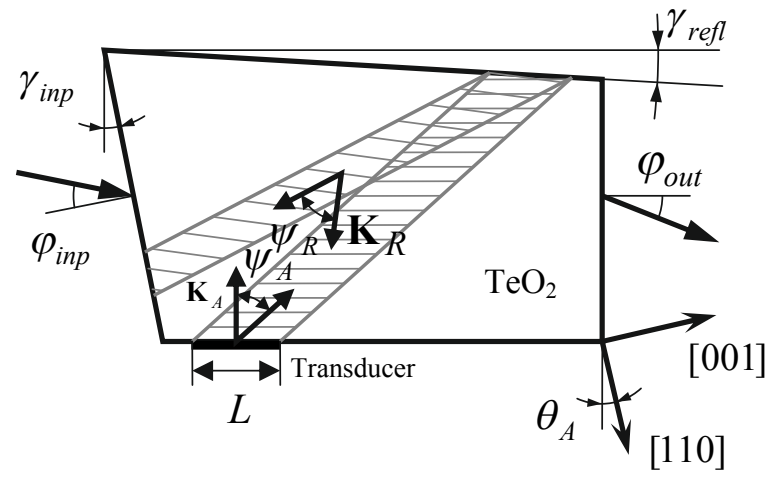

Fig. 2. Construction of our device utilizing $A O$ interaction with the acoustic wave reflected from crystal boundary.

A transducer excites the shear wave polarized along the [1 $\overline{1} 0]$ direction. The wave front of the excited acoustic wave is parallel to the crystal face to which the piezoelectric transducer is attached, so that the direction of the wave vector $\mathbf{K}_{A}$ is fixed by the orientation of its bottom face. The shear wave propagating in the crystal in this geometry suffers complete reflection at the top boundary, with no mode conversion, because its polarization is perpendicular to the plane of incidence and coincides with the polarization of the same eigenmode after reflection, while the polarizations of the other modes have zero projections along this direction. Of course, there are also subsequent reflections of the acoustic wave from other crystal faces. However, we do not consider the multiple acoustic reflections as they produce no undesirable diffraction effects. 
The direction of the reflected wave vector $\mathbf{K}_{R}$ is defined by the inclination angle $\gamma_{\text {refl }}$ of the top crystal face, which should be properly selected to match the Bragg conditions and enable diffraction at the reflected wave. Thus, the angles $\theta_{A}$ and $\gamma_{\text {refl }}$ represent important construction parameters, which fix the orientations of the direct (forward) and reflected (backward) acoustic waves inside the crystal and cannot be changed after the AO cell is manufactured. If the inclination angle $\gamma_{i n p}$ of the input face is selected in a proper manner, it becomes possible to make the directions of the input light entering the crystal and the output light leaving the device coincide. We denote the orientations of the input and output light waves relative to the normals of the faces as $\varphi_{\text {inp }}$ and $\varphi_{\text {out }}$, respectively.

As a starting point for determining all the relevant construction angles, we have used our standard AO-cell parameters: the orientation angle of the ultrasound wave vector $\theta_{A}=7.90^{\circ}$ and the inclination of the input face $\gamma_{i n p}=3.68^{\circ}$. We choose to keep these angles invariable, because we have already had a suitable experimental sample. Instead, we will optimize all of the other angles. These are the angle $\gamma_{\text {refl }}$ that defines inclination of the reflecting face, as well as the internal angles of incidence $\left(\theta_{i}\right)$ and diffraction $\left(\theta_{d}\right)$.

As stressed before, the wave vectors of the acoustic waves propagating in paratellurite are usually selected to be close to the [110] axis, which allows for obtaining a high diffraction efficiency with the slow shear wave. This applies also to the case of reflected acoustic wave. We try to select the light propagation direction not too close to the optic axis, in order to make the ellipticity of the optical eigenmodes small. Hence, a linear polarization of the interacting light waves can be assumed further on.

Having the construction angle $\theta_{A}$ fixed, we have performed a numerical search for the possible $\theta_{d}$ and $\theta_{R}$ values by varying the angle $\theta_{i}$ of light incidence in the range $0^{\circ} \ldots 20^{\circ}$. Solving the system of Eqs. (1), (4) and (7) for each of the $\theta_{i}$ values and assuming that $\theta_{A}$ is constant, we have found the vector length $\left|\mathbf{K}_{A}\right|$ required for satisfying the Bragg conditions for the "oe" diffraction process. This gives us the diffraction angle value $\theta_{d}$. Switching to the process of "eo" diffraction and using the $\theta_{i}$ and $\theta_{d}$ values obtained at the previous step, we have found the direction $\theta_{R}$ and the length $\left|\mathbf{K}_{R}\right|$ of the reflected acoustic wave required for satisfying the Bragg conditions. The corresponding frequencies necessary for driving the piezoelectric transducer can easily be found from the lengths of wave vectors for the direct and reflected waves, using Eq. (3). The resulting plots for the $\theta_{d}$ and $\theta_{R}$ angles and the driving frequencies, $f_{A}=\Omega_{\mathrm{A}} / 2 \pi$ and $f_{R}=\Omega_{\mathrm{R}} / 2 \pi$, are presented in Fig. 3 .
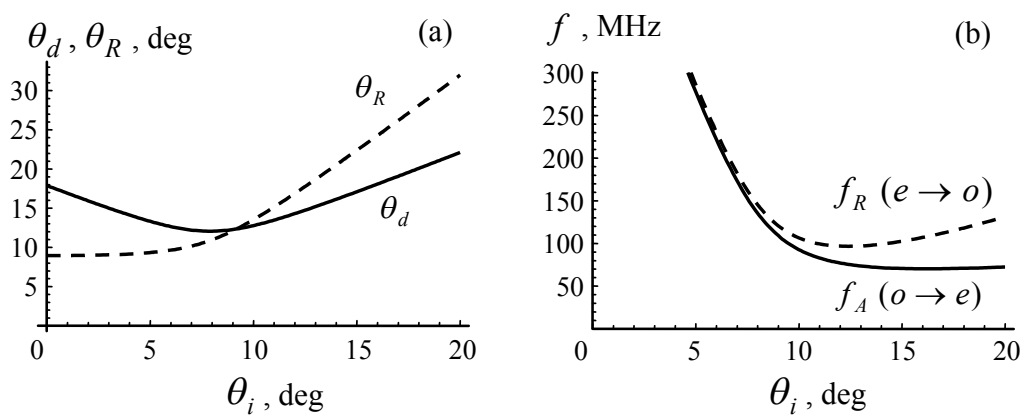

Fig. 3. Calculated dependences of diffraction parameters on the propagation angle of incident light: (a) required directions of reflected acoustic wave and expected diffraction angles; (b) driving frequencies required to obtain the diffraction.

Ukr. J. Phys. Opt. 2018, Volume 19, Issue 2 
Now we are to decide which angle, out from the set of possible $\theta_{i}$ values, should be used in our actual device. The typical ultrasound-frequency range used in our AO device is $60-110 \mathrm{MHz}$, which makes the angles $\theta_{i}>9.7^{\circ}$ favourable (see the frequency plots in Fig. 3b). On the other hand, it would be better to use the smallest possible angle $\theta_{R}$ for the acoustic wave, since the ultrasound velocity is lower near the [110] axis, which facilitates higher diffraction efficiency. Smaller $\theta_{R}$ 's are achievable at smaller $\theta_{i}$ 's. As a result, we have decided to use the value $\theta_{i}=9.97^{\circ}$. This incident-light angle corresponds to the direction $\theta_{R}=13.49^{\circ}$ for the reflected acoustic wave. The group-velocity walk-off for the acoustic wave propagating in this direction is equal to $\psi_{R}=57.02^{\circ}$, while the walk-off angle for the direct acoustic wave is $\psi_{A}=50.64^{\circ}$.

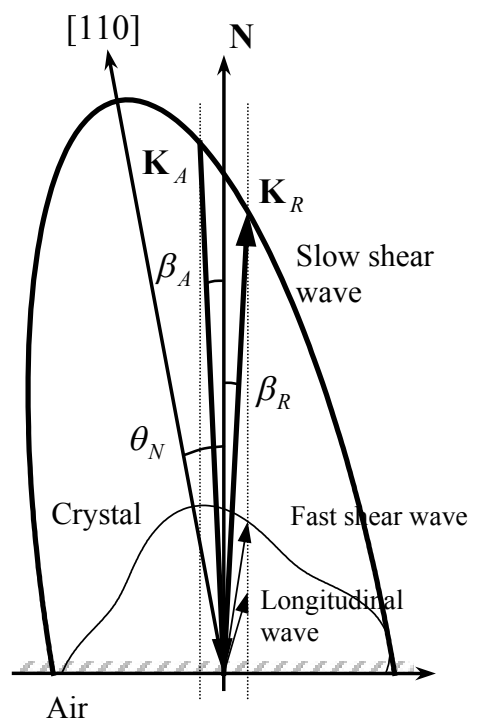

Fig. 4. Acoustic-wave reflection geometry obtained following from the slowness curves.

The required inclination angle $\gamma_{\text {refl }}$ of the reflecting face has been calculated using an anisotropic equivalent of the Snell's law from the slowness surfaces [13]. The corresponding reflection geometry and the slowness curves are shown in Fig. 4. The resulting angle between the top-face normal $\mathbf{N}$ and the [110] axis amounts to $\theta_{N}=10.50^{\circ}$, the angles of the incident and reflected wave vectors relative to the boundary normal are respectively $\beta_{A}=2.60^{\circ}$ and $\beta_{R}=2.99^{\circ}$, whereas the reflecting face inclination is given by $\gamma_{\text {refl }}=\beta_{A}=2.60^{\circ}$.

\section{Refined calculations of interaction geometry}

Using the directions of the acoustic wave vectors given by $\theta_{A}=7.90^{\circ}$ and $\theta_{R}=13.49^{\circ}$, we might have expected to obtain the diffraction with the incident light propagating at the angle $\theta_{i}=9.97^{\circ}$ and the diffracted light propagating at the angle $\theta_{d}=12.75^{\circ}$. However, these values have been estimated in the assumption that the light directions inside the crystal are the same for the both polarizations. As noted in Section 2, this is not true in general, and the actual light propagation directions inside the crystal are slightly different because of refraction at the input face. To make sure that the diffraction can still happen in our device, we have performed the additional calculations. This time we have taken into consideration birefringent refractions on both the input and output faces of the crystal. In these calculations, the angles $\theta_{A}$ and $\theta_{R}$ are fixed, and the angle $\varphi_{\text {inp }}$ for the light entering the crystal is varied in the range $-5^{\circ} \ldots 20^{\circ}$. The plots for the 
output angle for different polarizations are presented in Fig. 5a. For the same input angle $\varphi_{\text {inp }}$, the light diffracted at the direct ultrasound wave leaves the crystal at the angle $\varphi_{\text {out }}$, while the light of different polarization which diffracts at the reflected ultrasound wave leaves the crystal at the angle $\varphi_{\text {outR }}$.
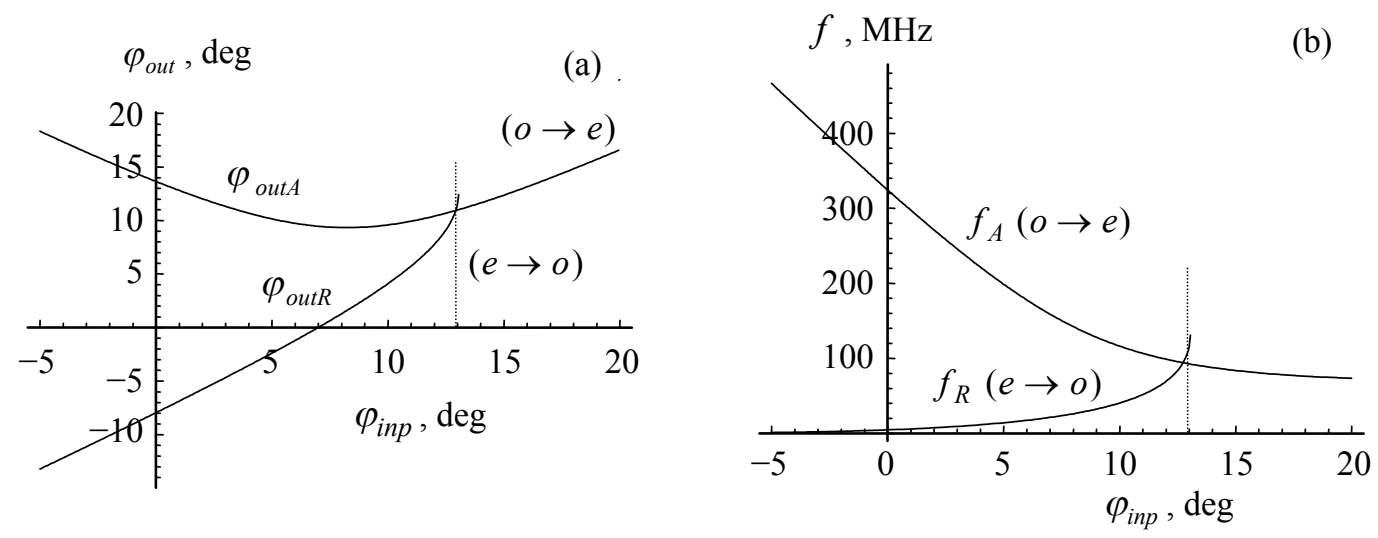

Fig. 5. Calculated input-angle dependences of (a) angle at which the light of different polarizations leaves the crystal and (b) driving frequencies required to obtain the diffraction.

Our calculations have shown that these two angles become equal to each other at $\varphi_{\text {inp }}=12.928^{\circ}$, so that we arrive at $\varphi_{\text {outA }}=\varphi_{\text {out }}=10.920^{\circ}$. At the same time, the internal angles of incident-light propagation inside the crystal are slightly different for different polarizations, $\theta_{i o}=9.979^{\circ}$ and $\theta_{i e}=9.968^{\circ}$. The diffraction angles are also different, being equal to $\theta_{d e}=12.762^{\circ}$ and $\theta_{d o}=12.777^{\circ}$. Nevertheless, the both diffracted beams leave the crystal in the same direction due to the birefringence. The plots for the driving frequency required to obtain the diffraction for one or the other polarization are shown in Fig. 5b. These frequencies amount to $f_{A}=93.02 \mathrm{MHz}$ and $f_{R}=107.55 \mathrm{MHz}$ for the case of $\varphi_{\text {inp }}$ angle selected above.

Hence, our calculations have confirmed that the error introduced initially by ignoring the refraction at the input face is indeed small, and the angular difference for different polarizations caused by the refraction at the input and output crystal faces can easily be compensated by finely tuning the input angle of light. Moreover, the plots presented in Fig. 5 show that the diffraction at the reflected acoustic wave is much more sensitive to the angular and frequency misalignments, if compared with the diffraction happening at the direct acoustic wave.

\section{Parallax compensation}

In the device presented above, the diffraction angles for different light polarizations are very close to each other but, nevertheless, the light travels along slightly different paths inside the crystal. Due to this fact, the light of different polarizations can leave the crystal at different points, thus leading to an undesirable parallax effect. The path difference comes from the two reasons. The first is related to different locations of virtual centres at which the diffraction takes place. The second reason is the group-velocity walk-off for the optical rays (see Fig. 6).

While the wave-vector directions of the waves with different polarizations are almost the same, the directions of group velocities for the extraordinary rays are noticeably skewed due to anisotropy of the material medium. The group-velocity walk-off for the optical rays can be 


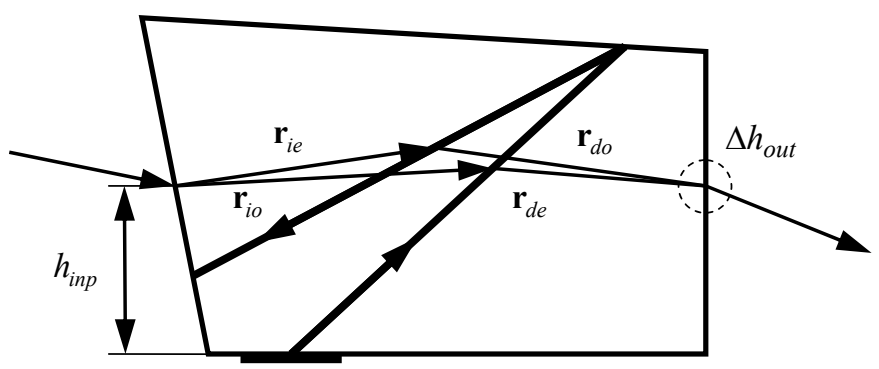

Fig. 6. Compensation of output parallax by selecting optimal height of light entrance.

estimated using Eq. (5), where the velocity should be replaced with the inverse refraction index. In the configuration selected by us, the walk-off angle for the incident extraordinary ray $\mathbf{r}_{i e}$ is equal to $\delta_{i e}=1.179^{\circ}$, while for the diffracted ray $\mathbf{r}_{d e}$ it is even larger, $\delta_{d e}=1.493^{\circ}$. Still, it is possible to compensate the total path difference inside the crystal via selecting properly the entry-point position $h_{\text {inp }}$, thus making the exit points coincide for different light polarizations. Tracing the light rays diffracted at the acoustic wave as a result of simple ray bending at the intersection points gives an estimate of the optimal position, $h_{i n p}=7 \mathrm{~mm}$.

\section{Experimental verification}

A paratellurite sample, from which the AO cell was made, had the dimensions $17 \times 10 \times 10 \mathrm{~mm}^{3}$. The piezoelectric transducer made of a $163^{\circ} \mathrm{Y}$-cut $\mathrm{LiNbO}_{3}$ was attached to the bottom face at the distance $1 \mathrm{~mm}$ from the left edge, using a cold vacuum welding (see Fig. 2). It had the size $L \times H=4 \times 8 \mathrm{~mm}^{2}$. The transducer was oriented so that to excite the shear acoustic waves polarized along the $[1 \overline{1} 0]$ direction. The orientation of the bottom face $\left(\theta_{A}=7.90^{\circ}\right)$ and the inclination of the input face $\left(\gamma_{i n p}=3.68^{\circ}\right)$ were regular for our device, while the top reflecting face was inclined at the angle $\gamma_{\text {reff }}=2.60^{\circ}$, in accordance with the calculations presented above.

The experimental measurements were conducted with a $785 \mathrm{~nm}$ laser source emitting linearly polarized light. The polarization was oriented at $45^{\circ}$ with respect to the incidence plane, thus making the intensities of optical eigenmodes inside the crystal equal to each other. The light of one or the other polarization appeared at the output of the device depending on the driving frequency applied to the transducer. The driving frequencies were adjusted so that to make the light of different polarizations leave the crystal at the same angle. The exact frequencies used in our experiments to make this happen were $94.3 \mathrm{MHz}$ for the "oe" diffraction and $108.0 \mathrm{MHz}$ for the "eo" diffraction. These values are close to the calculated values, whereas the small difference can possibly be explained by manufacturing tolerances. The parallax compensation was achieved for the beam shift $h_{\text {inp }}=6.5 \mathrm{~mm}$, which is also close to the value estimated theoretically. During parallax verification, we used a narrow laser beam having the approximate diameter $1 \mathrm{~mm}$.

The diffraction efficiency achieved experimentally was different for different polarizations, when the same electrical power $(800 \mathrm{~mW})$ was applied to the transducer. The ratios of the diffracted light intensity to the input intensity were equal to $I_{\text {out }} / I_{0}=40 \%$ and $32 \%$ for the "oe" and "eo" diffractions, respectively. The corresponding field structure of the diffracted light at the output of our device detected with a wide laser beam is depicted in Fig. 7. The difference between diffraction efficiencies for the two polarizations appears due to different AO figures of merit for 
the direct and reflected acoustic waves, and also because of different interaction lengths originating from the changes in the acoustic-beam size after reflection.

The calculations demonstrate that the greatest influence on the figure of merit $M_{2}$ $\left(M_{2}=n^{6} p_{e f f}^{2} / \rho V^{3}[10]\right)$ comes from the change in the ultrasound velocity, while all the other parameters, including the effective photoelastic constant $p_{\text {eff }}$, are approximately the same for the both diffraction modes. The ultrasound velocities of the direct and reflected waves are $V\left(\theta_{A}\right)=672 \mathrm{~m} / \mathrm{s}$ and $V\left(\theta_{R}\right)=772 \mathrm{~m} / \mathrm{s}$. Therefore the ratio of the AO figures of merit is $M_{2}^{o e} / M_{2}^{e o} \approx V\left(\theta_{R}\right)^{3} / V\left(\theta_{A}\right)^{3} \approx 1.52$, while the ratio of the diffracted intensities obtained experimentally is 1.41 , which is quite close to our theoretical estimation. Notice that our measurements have been performed in a weak-interaction mode, when the ultrasound power is low enough to minimize the effects of nonlinearity of intensity changes, which can appear at high ultrasound powers. In any practical device, the lower intensity of the "eo" diffraction can easily be compensated by applying higher electric powers at the driving frequency 108.0 MHz. This has been tested experimentally and allowed achieving the diffraction efficiency $40 \%$ for the both polarizations.

Direct acoustic wave

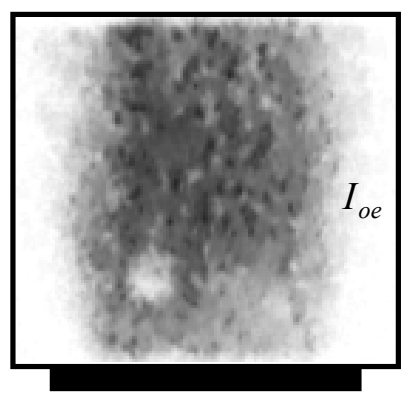

Transducer
Reflected acoustic wave

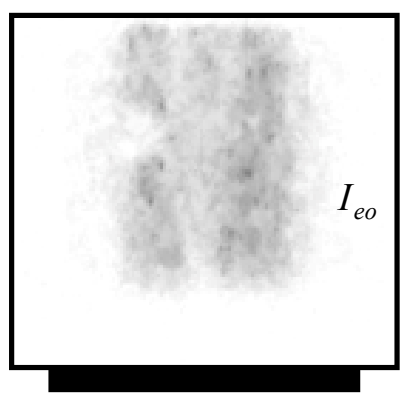

Transducer

Fig. 7. Distributions of light intensities at the output face of our device, as obtained from the cases of diffraction at the direct and reflected acoustic waves.

The contrast ratio of our device is given by

$$
C R=\frac{I_{\max }-I_{\min }}{I_{\min }},
$$

where $I_{\max }$ and $I_{\min }$ are the intensities of useful and parasitic components of the output light. The parasitic component of light passing through the device is associated with the ellipticity of eigenmodes propagating inside the crystal and the occurrence of undesirable polarization component at the device output, though one expects the output light to be perfectly linearly polarized in the ideal case.

The maximal contrast ratio achievable theoretically can be estimated as $C R_{\max }=1 / \xi^{2}-2 / \xi$, where the ellipticity $\xi$ of the optical wave is given by Eq. (11). The ellipticity of the incident light amounts to $\xi_{i}=0.0271$, while the ellipticity of the diffracted light is somewhat smaller, $\xi_{d}=0.0165$. This is because the diffracted light propagates at larger angles with respect to the optic axis. Only the ellipticity of the diffracted light that leaves the crystal is of 
importance in a practical case, since the useful light comes always from the diffracted wave. Therefore the maximal achievable contrast ratio is $C R_{\max }=1 / \xi_{d}^{2}-2 / \xi_{d}=3552: 1$.

To measure experimentally the contrast ratio of the device operating as a polarization switch, an additional polarizer was placed between the output of the device and the photodetector. The intensities of the parasitic and useful components were measured at the crossed and parallel polarizer orientations. Note that, in the latter case, the polarizer orientation is parallel to the expected dominant polarization direction. The measured contrast ratio was equal to $C R \approx 1800: 1$. We suppose that the contrast ratio achieved experimentally is limited not only by the ellipticity of eigenmodes, but also by some other factors like the light scattering at the impurities available in the crystal. This explains the difference of the measured and predicted contrast ratios.

The time response of our device depends on how fast a given polarization is switched to its alternative after the driving frequency at the piezoelectric transducer has been changed. The switching time is determined by the acoustic-wave velocities. It is not larger than $14 \mu \mathrm{s}$ and $22 \mu \mathrm{s}$ respectively for the " $o e$ " and "eo" diffractions.

\section{Conclusions}

The AO switch of laser-beam polarization based on the anisotropic Bragg diffraction in paratellurite has been suggested and investigated experimentally. The device is constructed so that the diffraction conditions are satisfied for the two optical modes simultaneously. The acoustic wave reflected from the crystal face is used to obtain the required direction of ultrasound propagation. The complete procedure for calculating the parameters of the device has been outlined. The device can perform switching (or modulation) of the output light polarization, which is governed by changes in the driving frequency of acoustic signal applied to the piezoelectric transducer. The construction of the device suggested by us enables compensating the parallax effect, provided that narrow laser beams are used.

The experimental verification has confirmed a possibility for constructing the polarization switch with the high contrast ratio, 1800:1, using a single piezoelectric transducer. The efficiency of diffraction at the direct acoustic wave is higher than that associated with the reflected wave, whenever the same driving power is applied to the transducer. The reason is the difference in the corresponding AO figures of merit. In any practical case, lower diffraction efficiencies can easily be compensated by applying higher electrical powers to the ultrasound transducer.

\section{Acknowledgements.}

This work was supported by the Ministry of Education and Science of Ukraine (under the Project No. 16BF052-04/0116U002564).

\section{References}

1. Antonov SN and Mirgorodsky V I, 2004. Anisotropic acoustooptic modulator of nonpolarized light based on diffraction by a slow acoustic wave in a paratellurite crystal. Techn. Phys. 49: 83-86.

2. Antonov S N, 2004. Acoustooptic nonpolar light controlling devices and polarization modulators based on paratellurite crystals. Techn. Phys. 49: 1329-1334.

3. Volochinov V and Polikarpova N, 2003. Collinear tunable acousto-optic filters applying acoustically anisotopic material tellurium dioxide. Molecular and Quantum Acoustics. 24: 225-235. 
4. Voloshinov V B and Polikarpova N V, 2009. Acousto-optic investigation of propagation and reflection of acoustic waves in paratellurite crystal. Appl. Opt. 48: C55-C66.

5. Uchida $\mathrm{N}$ and Ohmachi Yo, 1969. Elastic and photoelastic properties of $\mathrm{TeO}_{2}$ single crystal. J. Appl. Phys. 40: 4692-4695.

6. Yano T, Kawabuchi M, Fukumoto A and Watanabe A, 1975. $\mathrm{TeO}_{2}$ anisotropic Bragg light deflector without midband degeneracy. Appl. Phys. Lett. 26: 689-691.

7. Pilgun $\mathrm{Yu} \mathrm{V}$ and Smironov Ye M, 2010. Broadband acousto-optic diffraction of twowavelength light in paratellurite. Ukr. J. Phys. Opt. 11: 28-43.

8. Ohmachi Yo and Uchida N, 1970. Temperature dependence of elastic, dielectric, and piezoelectric constants in $\mathrm{TeO}_{2}$ single crystals. J. Appl. Phys. 41, 2307-2311.

9. Ohmachi Yo, Uchida $\mathrm{N}$ and Niizeki $\mathrm{N}, 1972$. Acoustic wave propagation in $\mathrm{TeO}_{2}$ single crystal. J. Acoust. Soc. Amer. 51: 164-168.

10. Yariv A. and Yeh P. Optical waves in crystals. New York: Wiley-Interscience Publ. (1987).

11. Uchida N, 1971. Optical properties of single-crystal paratellurite $\left(\mathrm{TeO}_{2}\right)$. Phys. Rev. B. 4: 3736-3745.

12. Yano $\mathrm{T}$ and Watanabe $\mathrm{A}, 1974$. Acousto-optic figure of merit of $\mathrm{TeO}_{2}$ for circularly polarized light. J. Appl. Phys. 45: 1243-1245.

13. Royer D. and Dieulesaint E. Elastic waves in solids. Part I. Berlin: Springer-Verlag (2000).

Boichuk N. A., Pilgun Yu. V. and Smirnov Ye. M. 2018. Acousto-optic polarization switch with parallax compensation. Ukr.J.Phys.Opt. 19: $69-79$

Анотація. Запропоновано акустооптичний (АО) перемикач поляризації лазерного світла, що базується на кристалі парателуриту. Геометрію анізотропної взаємодії обрано для одночасного виконання умов Брегта для двох альтернативних поляризаиій світла з довжиною хвилі 785 нм. Для иього використовуються пряма та відбита від грані кристала акустичні хвилі. Поляризацією вихідного світла керують, перемикаючи частоту ультразвукового сигналу, поданого на АО-комірку, між двома альтернативними значеннями. Пристрій компенсує паралакс, так що світлові промені з різними поляризачіями залишають комірку в тій самій точиі, без поперечного зсуву, та потирюються в одному напрямку. Відповідний поляризачійний АО-перемикач було виготовлено та досліджено експериментально. Виміряне значення коефічієнт контрастності пристрою сягає 1800:1. 